\title{
Breast Lesion Excision System in the diagnosis and treatment of intraductal papillomas - A feasibility study
}

\section{Niinikoski, Laura}

2018-01

Niinikoski , L , Hukkinen , K, Leidenius , M H K, Ståhls , A \& Meretoja , T J 2018 , ' Breast Lesion Excision System in the diagnosis and treatment of intraductal papillomas - A

feasibility study ' , European Journal of Surgical Oncology , vol. 44 , no. 1 , pp. 59-66 . https://doi.org/10.1016/j.ejso.

http://hdl.handle.net/10138/300143

https://doi.org/10.1016/j.ejso.2017.10.213

publishedVersion

Downloaded from Helda, University of Helsinki institutional repository.

This is an electronic reprint of the original article.

This reprint may differ from the original in pagination and typographic detail.

Please cite the original version. 


\title{
Breast Lesion Excision System in the diagnosis and treatment of intraductal papillomas - A feasibility study
}

\author{
Laura Niinikoski ${ }^{\text {a, }}$, Katja Hukkinen ${ }^{\text {b }}$, Marjut H.K. Leidenius ${ }^{\text {a }}$, \\ Anders Ståhls ${ }^{c}$, Tuomo J. Meretoja ${ }^{a}$ \\ ${ }^{a}$ Breast Surgery Unit, Comprehensive Cancer Center, University of Helsinki and Helsinki University Hospital, \\ P.O. Box 263, 00029 HUS, Finland \\ ${ }^{\mathrm{b}}$ HUS Medical Imaging Center, Radiology, University of Helsinki and Helsinki University Hospital, P.O. Box \\ 263, 00029 HUS, Finland \\ ${ }^{\mathrm{c}}$ Department of Pathology, University of Helsinki and HUSLAB, Helsinki University Hospital, P.O. Box 400, \\ 00029 HUS, Finland
}

Accepted 10 October 2017

Available online 14 November 2017

\begin{abstract}
Objectives: This study aims to evaluate the feasibility of Breast Lesion Excision System (BLES) in the treatment of intraductal papillomas. Material and methods: All patients with a needle biopsy -based suspicion of an intraductal papilloma who consequently underwent a BLES procedure at Helsinki University Hospital between 2011 and 2016 were included in this retrospective study. The purpose of the BLES procedure was either to excise the entire lesion or in few cases to achieve better sampling.

Results: In total, 74 patients underwent 80 BLES procedures. Pathological diagnosis after the BLES biopsy confirmed an intraductal papilloma without atypia in 43 lesions, whereas 10 lesions were upgraded to high-risk lesions (HRL) with either atypical ductal hyperplasia or lobular carcinoma in situ. Five cases were upgraded to malignancy, two were invasive ductal carcinomas and three were ductal carcinoma in situ. Additionally, 18 lesions were diagnosed as other benign lesions. Four procedures failed. Complete excision with BLES was achieved in 19 out of 43 intraductal papillomas, 6 out of $10 \mathrm{HRL}$ and two out of five malignant lesions. No major complications occurred. The BLES procedure was adequate in the management of the 71 breast lesions.

Conclusion: The BLES procedure is an acceptable method for the management of small benign and high-risk breast lesions such as intraductal papillomas in selected patients. Thus, a great amount of diagnostic surgical biopsies can be avoided.
\end{abstract}

(C) 2017 Elsevier Ltd, BASO The Association for Cancer Surgery, and the European Society of Surgical Oncology. All rights reserved.

\section{Introduction}

Clinical, radiological and histological interpretation of papillary lesions of the breast remains challenging due to their wide morphological spectrum. Papillomas may be broadly divided into two groups: peripheral and central.

Abbreviations: BLES, Breast Lesion Excision System; HRL, high-risk lesion; ADH, atypical ductal hyperplasia; DCIS, ductal carcinoma in situ; CNB, core needle biopsy; VACNB, vacuum-assisted core needle; FNAC, fine needle aspiration cytology; MDT, multidisciplinary team; LCIS, lobular carcinoma in situ.

* Corresponding author. Surgical Hospital, P.O. Box 263, 00029 HUS, Finland.

E-mail address: laura.niinikoski@hus.fi (L. Niinikoski).
Peripheral papillomas are often multiple and central are solitary. Papillomas may on the basis of the morphology be classified into benign or papillomas associated with atypical ductal hyperplasia (ADH) or papilloma associated with ductal carcinoma in situ (DCIS) or malignant subtype e.g. papillary carcinoma with adjacent invasive carcinoma. Papillomas presenting as papillomatosis confers a higher risk of malignancy [1,2].

It is not possible to exclude atypia nor malignancy by core needle biopsy (CNB) or imaging [2-5]. Thus, the standard of care in the management of papillomas is surgical excision. Intraductal papillomas without atypia could be managed by follow-up and a surgical procedure could be avoided [6]. However, follow-up can be stressful for the 
patient and is a notable expenditure too. It has been suggested that a larger biopsy sample might decrease the risk of sampling error [7-9].

The Breast Lesion Excision System (BLES; Intact ${ }^{\mathrm{TM}}$; Intact Medical Corporation, Framingham, USA) is a biopsy device, which uses radiofrequency cautery to excise a single spheroid tissue sample without fragmentation thus making the histopathological analysis more accurate than by CNB. In the USA, BLES was approved by the Food and Drug Administration for sampling in 2001 and for complete removal of lesions in 2005. The BLES technique has shown to be a safe and potential alternative to traditional surgical excision for removal of small breast lesions [10-14]. The underestimation rate seems to be lower with the BLES than with vacuum-assisted core needle biopsy (VACNB) $[15,16]$. Other benefits of the BLES in comparison to VACNB are the possibility to evaluate histological margins and the ability of the BLES wire basket to perform hemostasis. The BLES biopsy can be carried out as an outpatient procedure.

The aim of this study is to evaluate the feasibility of BLES in the treatment of intraductal papillomas in selected patients.

\section{Patients and methods}

\section{Patients}

All patients with a needle biopsy - based suspicion of an intraductal papilloma who consequently underwent a BLES procedure at the Department of Radiology of Helsinki University Hospital (HUH) between November 2011 and June 2016 were included in this retrospective study. The patients had a prior $\mathrm{CNB}$ or a fine needle aspiration cytology (FNAC) sample taken. In one BI-RADS 4 lesion, neither previous CNB nor FNAC was performed, but the lesion was biopsied with the BLES at the same session with another papilloma with a previous CNB. Only four of our patients had had nipple discharge. The purpose of the BLES was either to excise the entire lesion or in few cases to get a greater amount of tissue for better sampling of the lesion.

The patient data were collected into a database at the time of the BLES procedure. The HUH Department of Radiology is the first unit in Finland to use the BLES since 2011. The very first BLES procedures of our institute were also included in this series. At the beginning, four of the patients with a high-risk lesion (HRL) and positive margins in the BLES specimen were referred to surgical excision in order to confirm the diagnosis and a complete removal. The patients have been followed up since the first BLES procedure until June 2016. The procedure data was collected from the prospective database and additional data was gathered from electronic patient records retrospectively. The institutional research permission was granted by HUH Comprehensive Cancer Center.

\section{BLES procedure}

The BLES device consists of a biopsy basket, which is mounted into a wand. A wire basket emerges from the tip of the wand cutting and cauterizing the breast tissue with radiofrequency energy enveloping the target lesion. A single intact spherical tissue sample may then be removed. The basket sizes in our unit are $12 \mathrm{~mm}, 15 \mathrm{~mm}$ and $20 \mathrm{~mm}$. The aim was always to use the largest basket when possible (Fig. 1).

Contraindications for the BLES procedure were a cardiac pacemaker, pregnancy and breastfeeding. Anticoagulants such as clopidogrel and warfarin were recommended to be stopped one week prior to the procedure.

The maximum size of the lesions was $10 \mathrm{~mm}$ in breast ultrasound in order to achieve a complete excision. The thickness of the breast, i.e. the distance between the pectoralis muscle and the skin should be more than $14 \mathrm{~mm}$ so that the wire basket could open safely and successfully. The distance from the lesion to the skin and the pectoralis muscle should be $3 \mathrm{~mm}$ and to the nipple $6 \mathrm{~mm}$. Hereby, the most peripheral or most central papillomas are not feasible for the BLES.

The procedure was performed under ultrasound or stereotactical guidance by experienced senior breast radiologists. If residual lesion was seen, the procedure could be repeated. In case of two samples, the nipple's side of the specimen was inked so that the pathologist could better orientate for excision completeness. Clip mark was inserted into the biopsy site. The $1 \mathrm{~cm}$ skin incision was closed with strips. Total procedure time was approximately $50 \mathrm{~min}$.

BLES samples optimally fixed in $10 \%$ buffered formalin were sliced for processing, sectioning into $3 \mu \mathrm{m}$ thick sections and staining with hematoxylin and eosin according to approved methods and protocols in pathology laboratory. Samples were examined by pathologists specialized in breast pathology. In case of suspicion of e.g. ADH or malignancy adjunctive immunohistochemical stains were performed. The sections were stained with Ventana Benchmark XT (Roche, Ventana, Tucson, AZ), using biotin-free, three step multimer based detection kit Optiview (760-700, Roche, Ventana, Tucson, AZ). The protocol was based on heatinduced epitope retrieval using standard pretreatment buffer $\mathrm{CC} 1,64 \mathrm{~min}$. The slides were incubated for $40 \mathrm{~min}$ with the primary antibody to CK5/6, ER and SMMHC diluted 1:100. The slides were then dehydrated and mounted for viewing with microscope.

Histological margin status of the BLES sample was recorded and categorized into two groups: complete removal and partial removal (positive margin).

\section{Data analysis}

All cases were discussed at a multidisciplinary team (MDT) meeting after final histopathological diagnosis in order to decide upon definitive management: routine 

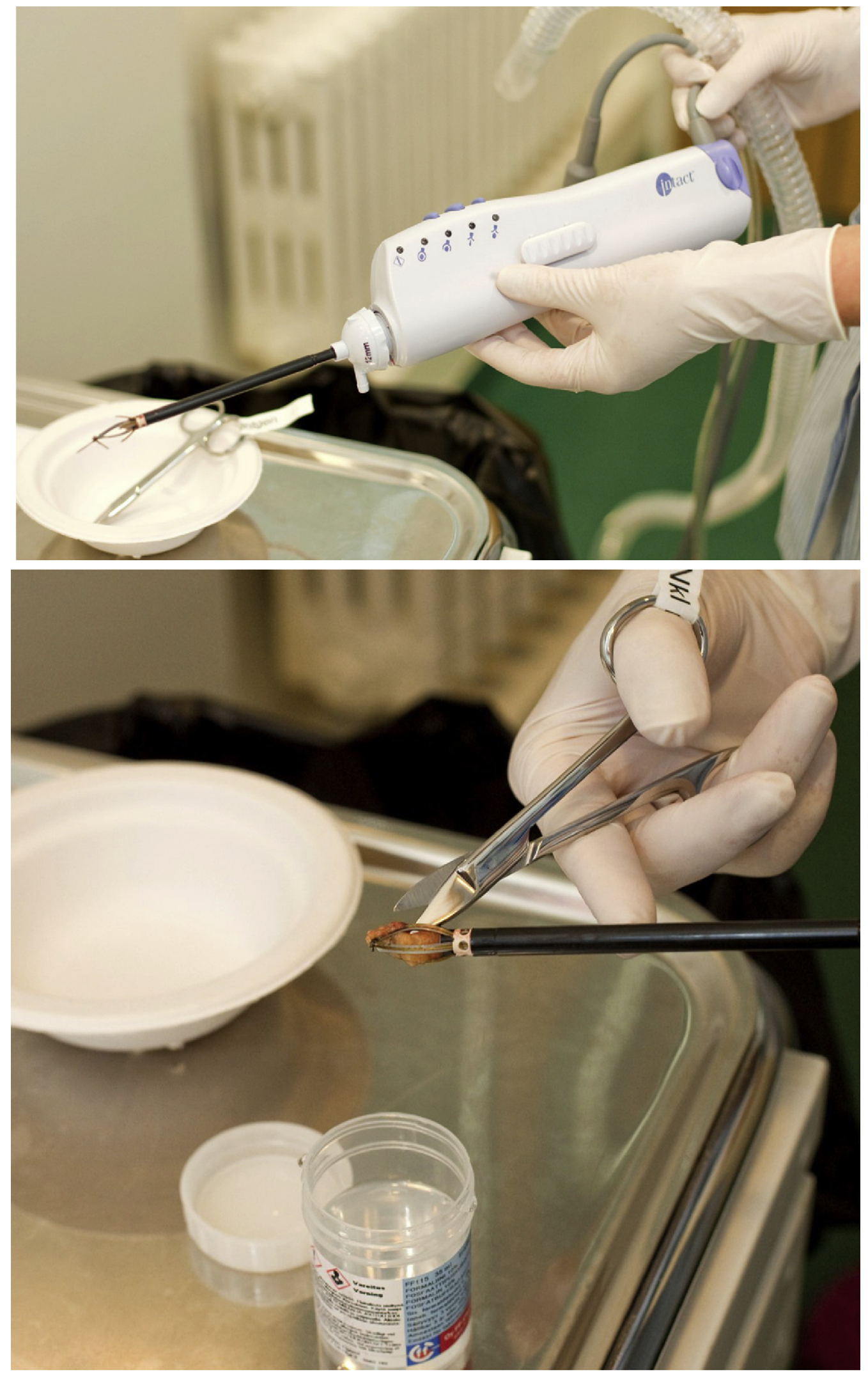

Fig. 1. The Breast Lesion Excision System (Intact ${ }^{\mathrm{TM}}$ ). 
screening, closer follow-up or further therapeutic surgery. In cases of further surgery, the surgical resection pathology was recorded as were the MDT recommendations after surgery. Follow-up imaging findings were gathered until June 2016 of those patients who were referred to our unit for the follow-up. The collected data included the size of the lesion on imaging, morphology and the BI-RADS classification of the lesion, the method of prior needle biopsy and the size of the wire basket used.

The pathological diagnosis of the BLES sample was compared with the needle biopsy result. We recorded the number of cases that were upgraded to a HRL such as ADH or lobular carcinoma in situ (LCIS), DCIS or to invasive carcinoma by the BLES. Percentage of complete removals was evaluated. We also looked at all the complications or difficulties related to the procedure. In addition, we performed a centralized re-evaluation of all samples with unclear pathology reports. An experienced breast pathologist re-evaluated these samples and presented a definitive interpretation.

\section{Results}

In total, 74 patients with a histological or cytological and radiological suspicion of an intraductal papilloma underwent 80 BLES procedures. The characteristics of the patients and the lesions are summarized in Table 1.

\section{Radiological findings}

$56(70 \%)$ of the lesions were circumscribed masses, 11 cystic and solid masses, eight irregular masses, four microcalcifications and one architectural distortion (Table 1). 67 (84\%) cases were classified as BI-RADS 4 and only two as BI-RADS 5.

\section{Histopathological findings}

Pathological diagnosis after the BLES biopsy confirmed an intraductal papilloma without atypia in 43 lesions (Table 2, Fig. 2). In total, 10 lesions were upgraded to HRL with either ADH or LCIS: Six lesions were diagnosed as intraductal papillomas with areas of ADH. Three cases were upgraded to ADH without an intraductal papilloma. One patient was found to have LCIS in addition to the papilloma.

Five cases were upgraded to malignancy, two of them had invasive ductal carcinomas and three had DCIS grade 2. All these lesions were BI-RADS 4: three circumscribed masses, one cystic and solid mass and one microcalcification.

The distribution of the lesion excision margins is presented in Tables 2 and 3. The median radiological size of the lesions which were excised completely was $7 \mathrm{~mm}$ $(4-12 \mathrm{~mm})$ whereas the mean size of the lesions excised partially was $9 \mathrm{~mm}(4-16 \mathrm{~mm})$. In both of the cases of invasive carcinomas, the margins were tumor-positive. For one of the DCIS cases, the data of the margin in the
Table 1

Characteristics of the patients, the procedure and the lesions.

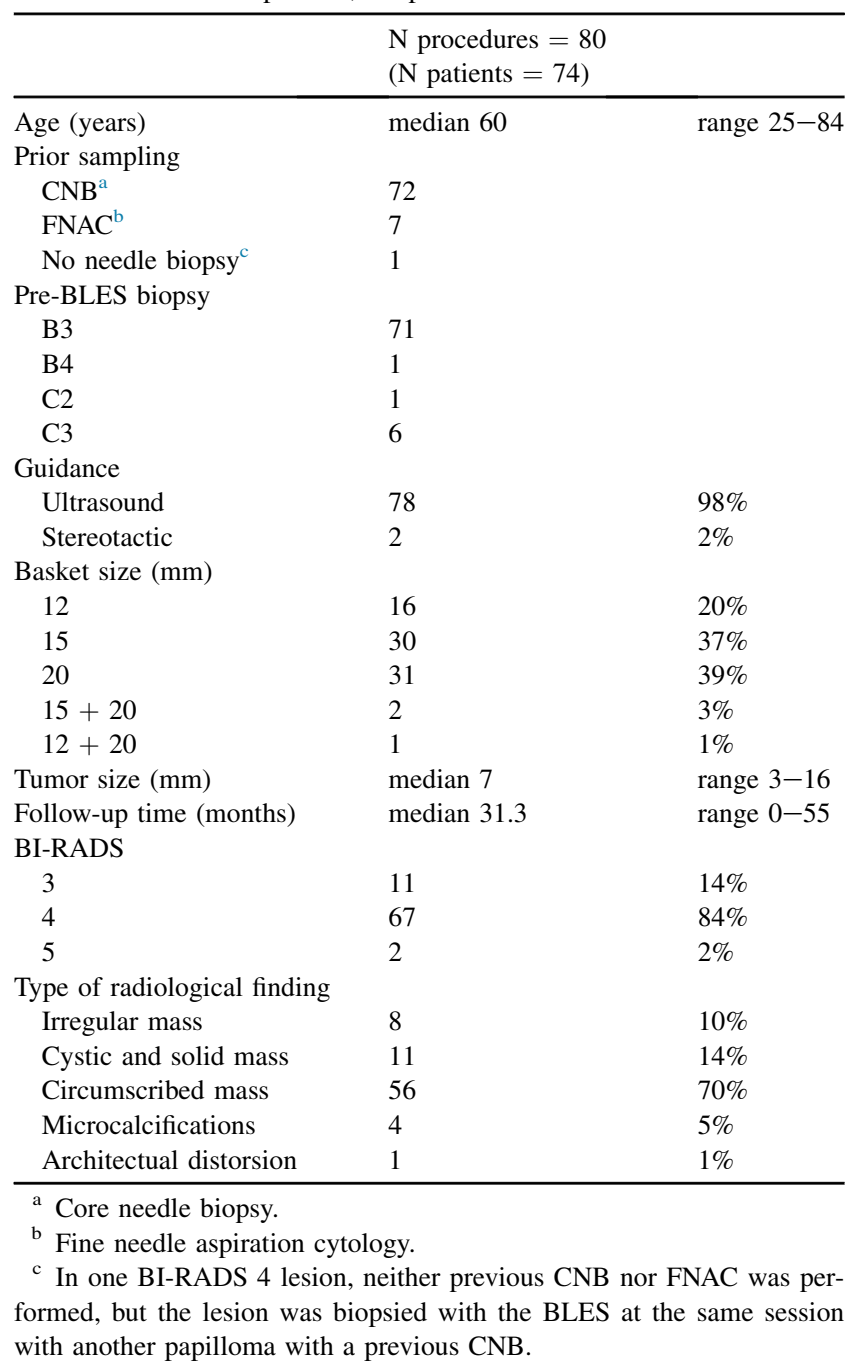

BLES sample was missing, but there was no residual DCIS found on the surgical specimen. We found no difference in the type of radiological abnormality with respect to the BLES sample margins.

In total, 18 lesions were diagnosed as other benign lesions (fibrocystic disease, fibrosis, adenosis or fibroadenoma). Three of them had a needle-biopsy based suspicion of an intraductal papilloma and ADH and thereby they were downgraded by BLES.

\section{Complications and challenges with the procedure}

Two $(2.5 \%)$ procedures failed technically. One procedure was unsuccessful due to small size of the breast and another one failed due to a hematoma which occurred after the injection of the anesthetic solution causing a loss of the visibility. These cases were biopsied surgically.

Two other cases were unsuccessful as a diagnostic procedure. In the other case the BLES indicated only fibrocystic disease, but since the patient had clear discharge 
Table 2

Histopathological findings and margins.

\begin{tabular}{lllll}
\hline & N procedures $=80(\mathrm{~N}$ patients $=74)$ & Complete excision & Partial excision & Unclear $^{\mathrm{a}}$ \\
\hline PAPILLOMAS & $\mathbf{4 3}$ & $\mathbf{1 9}(44.2 \%)$ & $\mathbf{2 4}(55.8 \%)$ & thermal damage in 2 \\
High-risk lesions & $\mathbf{1 0}$ & $\mathbf{6}(60 \%)$ & $\mathbf{4}(40 \%)$ & thermal damage in 1 \\
ADH $^{\mathrm{b}}+$ PAPILLOMA & 6 & 4 & 2 & thermal damage in 2 \\
ADH $^{\mathrm{b}}$ & 3 & 1 & 2 & \\
LCIS $^{\mathrm{c}}+$ PAPILLOMA & 1 & 1 & - & $1(20 \%)$ \\
Malignant & $\mathbf{5}$ & $\mathbf{2}(40 \%)$ & $\mathbf{2}(40 \%)$ & 1 \\
DCIS & 3 & 2 & 0 & $1(1.7 \%)$ \\
CARCINOMA & 2 & 0 & 2 & $30(51.7 \%)$ \\
\end{tabular}

a One sample with DCIS had unclear margin and presented as circumscribed mass on imaging.

b Atypical ductal hyperplasia.

${ }^{\mathrm{c}}$ Lobular carcinoma in situ.

${ }^{d}$ Ductal carcinoma in situ.

e All DCIS lesions were grade 2.

from the nipple a new ductography was performed and it still showed a suspicion of an intraductal papilloma. The patient underwent surgery and the surgical specimen revealed multiple papillomas and ADH. The other one was diagnosed as fibrocystic disease by BLES but due to radiological suspicion the patient went through a surgical excision with a final histological diagnosis of DCIS. This indicates that in these two cases the BLES basket did not manage to catch the right target.

In five histological report (6\%) there was a remark of thermal artefacts, none of which disturbed the histopathological analysis.

There were no complications that would have needed operative treatment. One hematoma turned into an abscess and was treated with oral antibiotics. Minor hematomas were not documented. Only one thermal injury of the skin occurred, it was excised under local anesthesia in the outpatient clinic right after the BLES procedure.

\section{Further surgery and follow-up}

Surgical removal was performed for all invasive carcinomas and DCIS in order to ensure negative histological margins. On surgical excision, no invasive carcinoma was found on specimen, only DCIS or ADH. Surgical biopsy was performed also in four of the intraductal papillomas and in one ADH case, in three of these cases there was residual papilloma tissue found on surgical specimen, but no $\mathrm{ADH}$.

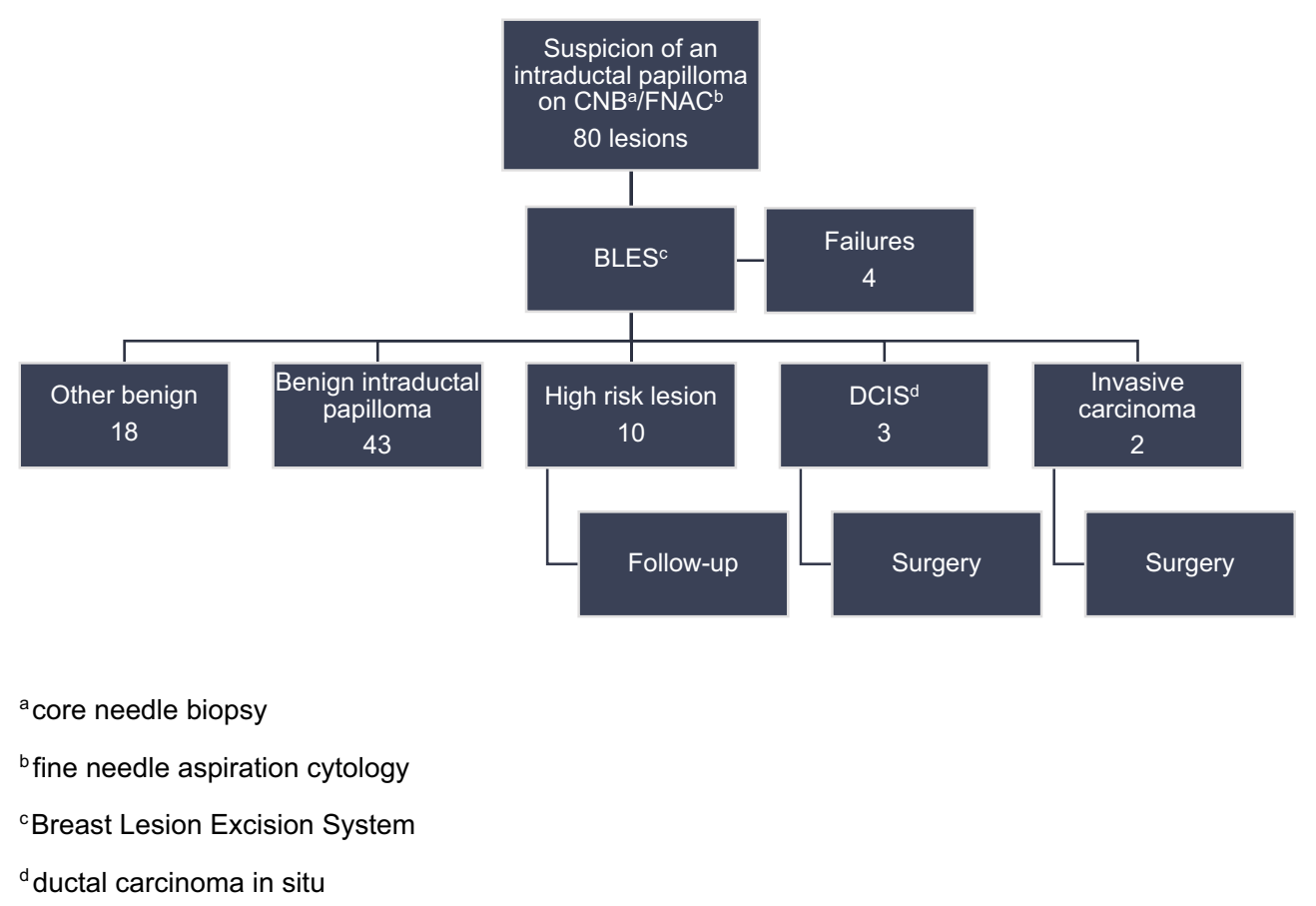

Fig. 2. Feasibility of the BLES procedure and distribution of histopathological findings. 
Table 3

Radiological abnormality and margins.

\begin{tabular}{lll}
\hline & $\begin{array}{l}\text { Complete excision } \\
\mathrm{N}=27\end{array}$ & $\begin{array}{l}\text { Partial excision } \\
\mathrm{N}=30\end{array}$ \\
\hline Size, median (range) & $7 \mathrm{~mm}(4-12 \mathrm{~mm})$ & $9 \mathrm{~mm}(4-16 \mathrm{~mm})$ \\
Type of radiological abnormality & \\
Circumscribed mass & $20(74.0 \%)$ & $20(66.7 \%)$ \\
Cystic and solid mass & $4(14.8 \%)$ & $5(16.7 \%)$ \\
Irregular mass & $2(7.4 \%)$ & $3(10.0 \%)$ \\
Microcalcification & $1(3.7 \%)$ & $1(3.3 \%)$ \\
Architectual distortion & - & $1(3.3 \%)$ \\
\hline
\end{tabular}

${ }^{\text {a }}$ One sample with DCIS had unclear margin and presented as circumscribed mass on imaging.

During the follow-up period, two patients have had a new intraductal papilloma in another location than the first one. Those were excised by BLES and they are included in this study. One patient has been diagnosed with a lobular carcinoma, it was found on the same side as the previous intraductal papilloma but in a different location. The rest of the patients have not had any papilloma recurrences.

\section{Discussion}

\section{BLES for benign and HRL}

This study reports a series of 80 BLES procedures for lesions with a suspicion of an intraductal papilloma. In our series, the BLES procedure was adequate in the management of 71 lesions (89\%) (Fig. 2). Thus, the BLES procedure rendered surgical excision obsolete in these patients. BLES can be carried out as a quick outpatient procedure and it is presumably more cost-effective than imageguided surgical biopsy requiring marking of non-palpable lesions beforehand. The cosmetic outcome of BLES procedure is good with just $1 \mathrm{~cm}$ scar. The procedure causes only moderate pain and no sick leave is needed.

No papilloma recurrences, HRL nor malignant tumors have been found during the follow-up in patients without surgical re-excision. The current treatment protocol of HRL in our unit is that there is no need for clear margins, it is sufficient to confirm the diagnosis with adequate sampling, i.e. with the BLES in selected cases. These patients are managed with follow-up. The standard care in patients with DCIS or invasive carcinoma is surgical excision.

There are several previous studies reporting on the use of BLES for sampling breast lesions but only few reports on performing complete excisions or using the BLES in the management of intraductal papillomas. Whitworth et al. [13] published a large series of 1170 patients who underwent a BLES for a mainly diagnostic purpose. They reported that none of the non-ADH lesions (lobular neoplasia, papilloma or radial scar) were upgraded to carcinoma on surgical excision or on radiological follow-up, so they suggested that there is no need of subsequent surgery. Seror et al. [11] studied the accuracy of BLES and the predictive factors for complete excision: $30 \%$ of the lesions were completely removed by BLES, and they were smaller in size. This is comparable with our findings.

Allen et al. [14] reported BLES to be an efficacious method in excising lesions smaller than $1 \mathrm{~cm}$. Among the 76 patients, they reported that 13 out of the 15 papillomas and all four ADH lesions were completely removed by BLES. In the other study by Allen et al. [10] 41 patients underwent BLES procedure in order to completely excise a high-risk or malignant lesion. The margins were clear for three out of the nine HRL and for 15 out of the 23 DCIS. None of the invasive carcinomas were completely removed. These results are also similar with ours.

There is a recent study from Scaperrotta et al. [17] using the BLES for small clusters of suspicious microcalcifications (BI-RADS 4) in 105 patients. They report that the BLES allows better histological interpretation and lower underestimation than VACNB, and they suggest it may have potential for a therapeutic role in selected patients.

There are also studies of using selective ductectomy for the management of intraductal papillomas. Selective ductectomy procedure requires that there is discharge coming from a duct and the duct can be cannulated. It is performed usually under general anesthesia and an incision of about one-third of the length of the areolar border is needed. Thus, selective ductectomy is more invasive procedure than BLES. However, unlike BLES, the procedure is suitable also for lesions that are close to the areola. A study of using selective ductectomy for the diagnosis and treatment of intraductal papillary lesions with single duct discharge by Maráz et al. [18] included 100 patients. They reported 6 cases of malignancy. Nine patients had ADH with papilloma and one patient had lobular neoplasia around the papilloma. This is comparable to our study.

\section{BLES risks and complications}

The present study confirms that BLES procedure is well tolerated as there were no major complications. Only one notable hematoma occurred, similarly to previous studies $[10-12,14]$. There were technical problems or insufficient sampling in a few cases but we want to highlight that none of the BLES cases were excluded despite a learning curve. In our series, one skin burn occurred; Al-Harathee et al. [19] reported two skin burns in their study which included 134 procedures. The only hematoma in our series turned into an infection. Sie et al. [16] reported one infection too but no other complications.

Thermal artefacts on BLES specimens have been reported in several studies $[11,12,15,17,20]$. However, it has been stated that thermal damage did not affect the diagnostic outcome. Our study supports these findings.

\section{Limitations and future aspects}

There was no standardized treatment protocol for BLES cases at our unit during the study period. The cases were 
discussed at the MDT and the definite management was decided individually. The follow-up period is rather short for some patients for making strong conclusions on recurrences.

There is a recently published study of using VACNB in the management of breast lesions [20]. However, the margin assessment is not feasible in VACNB specimen as the procedure removes the lesions piece by piece. Additionally, VACNB is not suitable for patients with an increased risk of bleeding since it does not perform hemostasis. On the other hand, with VACNB there are less limitations with respect to the location of the lesion; the BLES basket requires more space to open up.

That study by Strachan et al. [21] introduced a two-way protocol, one for lesions with atypia and one for lesions without atypia. Patients with atypia in CNB but not upgraded or downgraded by VACNB are offered 5-year mammographic follow-up. The patients without atypia in both CNB and in VACNB are discharged to routine screening.

However, there is a significant difference in the management of patients with a CNB diagnosis of a breast lesion without atypia but epithelial atypia found on VACNB; they were offered diagnostic surgical biopsy since with VACNB it is not possible to preserve the lesion architecture and differentiate ADH from DCIS, unlike with BLES. This is a clear advantage of the BLES method. Nevertheless, their two-way follow-up protocol seems rational and could be adopted in our unit with the BLES method.

The BLES procedure also has a potential for management of selected small carcinomas. Further clinical trials are required to assess whether it is possible to achieve clear margins in excising malignant lesions. The BLES device with $30 \mathrm{~mm}$ wire basket is already in use in the United States.

\section{Conclusion}

Breast imaging with more and more accurate imaging technology is detecting a growing number of small breast lesions leading to possible over diagnoses and over treatment since the majority of lesions have a very low risk of malignancy. The BLES procedure is an acceptable method for the management of small benign and high-risk breast lesions such as intraductal papillomas. Thus, a great amount of diagnostic surgical biopsies could be avoided.

\section{Role of the funding source}

The corresponding author was supported by research funds of the Helsinki University Hospital.

\section{Conflict of interest}

None.

\section{References}

[1] Tatarian T, Sokas C, Rufail M, Lazar M, Malhotra S, Palazzo JP, et al. Intraductal papilloma with benign pathology on breast core biopsy: to excise or not? Ann Surg Oncol 2016;23(8):2501-7.

[2] Shiino S, Tsuda H, Yoshida M, Jimbo K, Asaga S, Hojo T, et al. Intraductal papillomas on core biopsy can be upgraded to malignancy on subsequent excisional biopsy regardless of the presence of atypical features. Pathol Int 2015;65(6):293-300.

[3] Lam WW, Chu WC, Tang AP, Tse G, Ma TK. Role of radiologic features in the management of papillary lesions of the breast. AJR Am J Roentgenol 2006;186(5):1322-7.

[4] Rizzo M, Lund MJ, Oprea G, Schniederjan M, Wood WC, Mosunjac M. Surgical follow-up and clinical presentation of 142 breast papillary lesions diagnosed by ultrasound-guided core-needle biopsy. Ann Surg Oncol 2008;15(4):1040-7.

[5] Pareja F, Corben AD, Brennan SB, Murray MP, Bowser ZL, Jakate $\mathrm{K}$, et al. Breast intraductal papillomas without atypia in radiologic-pathologic concordant core-needle biopsies: rate of upgrade to carcinoma at excision. Cancer 2016 Sep 15;122(18): $2819-27$.

[6] Swapp RE, Glazebrook KN, Jones KN, Brandts HM, Reynolds C, Visscher DW, et al. Management of benign intraductal solitary papilloma diagnosed on core needle biopsy. Ann Surg Oncol 2013;20(6): 1900-5.

[7] Liberman L, Kaplan JB, Morris EA, Abramson AF, Menell JH, Dershaw DD. To excise or to sample the mammographic target: what is the goal of stereotactic 11-gauge vacuum-assisted breast biopsy? AJR Am J Roentgenol 2002 Sep;179(3):679-83.

[8] Darling ML, Smith DN, Lester SC, Kaelin C, Selland DL, Denison CM, et al. Atypical ductal hyperplasia and ductal carcinoma in situ as revealed by large-core needle breast biopsy: results of surgical excision. AJR Am J Roentgenol 2000 Nov;175(5):1341-6.

[9] Pandelidis S, Heiland D, Jones D, Stough K, Trapeni J, Suliman Y. Accuracy of 11-gauge vacuum-assisted core biopsy of mammographic breast lesions. Ann Surg Oncol 2003 Jan-Feb;10(1):43-7.

[10] Allen SD, Osin P, Nerurkar A. The radiological excision of high risk and malignant lesions using the INTACT breast lesion excision system. A case series with an imaging follow up of at least 5 years. Eur J Surg Oncol 2014;40(7):824-9.

[11] Seror JY, Lesieur B, Scheuer-Niro B, Zerat L, Rouzier R, Uzan S. Predictive factors for complete excision and underestimation of one-pass en bloc excision of non-palpable breast lesions with the Intact ${ }^{\circledR}$ breast lesion excision system. Eur J Radiol 2012;81(4):719-24.

[12] Medjhoul A, Canale S, Mathieu MC, Uzan C, Garbay JR, Dromain C, et al. Breast lesion excision sample (BLES biopsy) combining stereotactic biopsy and radiofrequency: is it a safe and accurate procedure in case of BIRADS 4 and 5 breast lesions? Breast J 2013 Nov-Dec;19(6):590-4.

[13] Whitworth PW, Simpson JF, Poller WR, Schonholz SM, Turner JF, Phillips RF, et al. Definitive diagnosis for high-risk breast lesions without open surgical excision: the Intact Percutaneous Excision Trial (IPET). Ann Surg Oncol 2011 Oct;18(11):3047-52.

[14] Allen SD, Nerurkar A, Della Rovere GU. The breast lesion excision system (BLES): a novel technique in the diagnostic and therapeutic management of small indeterminate breast lesions? Eur Radiol 2011 May;21(5):919-24.

[15] Killebrew LK, Oneson RH. Comparison of the diagnostic accuracy of a vacuum-assisted percutaneous intact specimen sampling device to a vacuum-assisted core needle sampling device for breast biopsy: initial experience. Breast J 2006 Jul-Aug;12(4):302-8.

[16] Sie A, Bryan DC, Gaines V, Killebrew LK, Kim CH, Morrison CC, et al. Multicenter evaluation of the breast lesion excision system, a percutaneous, vacuum-assisted, intact-specimen breast biopsy device. Cancer 2006 Sep 1;107(5):945-9. 
[17] Scaperrotta G, Ferranti C, Capalbo E, Paolini B, Marchesini M, Suman L, et al. Performance and role of the breast lesion excision system (BLES) in small clusters of suspicious microcalcifications. Eur J Radiol 2016 Jan;85(1):143-9.

[18] Maráz R, Boross G, Ambrózay É Svébis M, Cserni G. Selective ductectomy for the diagnosis and treatment of intraductal papillary lesions presenting with single duct discharge. Pathol Oncol Res 2013;19(3):589-95.

[19] Al-Harethee W, Theodoropoulos G, Filippakis GM, Papapanagiotou I, Matiatou M, Georgiou G, et al. Complications of percutaneous stereotactic vacuum assisted breast biopsy system utilizing radio frequency. Eur J Radiol 2013 Apr;82(4):623-6.

[20] Diepstraten SC, Verkooijen HM, van Diest PJ, Veldhuis WB, FernandezGallardo AM, Duvivier KM, et al. Radiofrequency-assisted intact specimen biopsy of breast tumors: critical evaluation according to the IDEAL recommendations. Cancer Imaging 2011 Dec 28;11:247-52.

[21] Strachan C, Horgan K, Millican-Slater RA, Shaaban AM, Sharma N. Outcome of a new patient pathway for managing B3 breast lesions by vacuum-assisted biopsy: time to change current UK practice? J Clin Pathol 2016 Mar;69(3):248-54. 